medgen $2012 \cdot 24: 253-256$

DOI 10.1007/s11825-012-0350-z

Online publiziert: 15. Dezember 2012

c) Springer-Verlag Berlin Heidelberg 2012
B. Wollnik · U. Kornak

Institut für Medizinische Genetik und Humangenetik, Charité - Universitätsmedizin Berlin

\section{Progeroide Erkrankungen und ihre Mechanismen}

Er fällt wegen seines schleichenden Verlaufs im Alltag kaum auf, kann aber z. B. beim Ansehen alter Fotos umso bewusster werden: der menschliche Alterungsprozess. In jedem menschlichen Gewebe kommt es im Lauf der Zeit zu typischen altersabhängigen Veränderungen, welche individuell in unterschiedlicher Kombination und zu verschiedenen Zeitpunkten auftreten können. Progeroide Erkrankungen bilden diesen komplexen Prozess niemals vollständig im Zeitraffer nach, sondern in einer unterschiedlich großen Anzahl von Geweben, weshalb man auch von segmentalen progeroiden Erkrankungen spricht [1]. Eine segmentale progeroide Erkrankung liegt per definitionem dann vor, wenn mindestens 2 Gewebe typische alterungsähnliche Veränderungen aufweisen. Unimodal progeroide Erkrankungen hingegen zeigen nur in einem Gewebe Anzeichen vorzeitiger Alterung (z. B. bei erblichen Formen der Parkinson-Erkrankung).

\section{》) Eine segmentale progeroide Erkrankung liegt vor, wenn mindestens 2 Gewebe alterungsähnliche Veränderungen aufweisen}

Oft ist zusätzlich ein progeroides Erscheinungsbild wichtiger Anlass, über eine Einordnung in diese Familie von Erkrankungen nachzudenken. Viele dieser Syndrome folgen einem monogenen Vererbungsmodus. Die Aufdeckung der zugrunde liegenden Gendefekte und ihrer Funktion hat wichtige Einsichten in die Mechanismen und Signalwege zutage gefördert, die den Verlauf der menschlichen Alterung beeinflussen [2]. Um diese besser einzuordnen, werden im Folgenden die momentanen Vorstellungen über Ursachen und evolutionäre Bedeutung des Alterns kurz zusammengefasst.

\section{Alterung und Gewebehomöostase}

Im Gegensatz zu Maschinen, deren Benutzung unweigerlich zum stetigen Verschleiß führt, dem nur durch Ersatzteile beizukommen ist, haben menschliche Gewebe ein immanentes Regenerationspotenzial. Warum reicht diese Regenerationsfähigkeit nicht aus, um die Gewebe dauerhaft in einer ausgewogenen Homöostase und ergo jung zu erhalten?

- Als einfachste Erklärung spielt es aus der Sicht der „selbstsüchtigen Gene“ keine Rolle, was nach erfolgter Reproduktion und Aufzucht der Nachkommen mit dem elterlichen Soma passiert, sodass der evolutionäre Druck zur Instandhaltung abnimmt [3].

- Die Disposable-Soma-Theorie von Kirkwood geht noch darüber hinaus und postuliert eine Umverteilung der limitierten Lebensenergie von der Regeneration in die Reproduktion als bestimmenden Faktor der Alterung [4].

- Eine dritte Sichtweise geht davon aus, dass Alterung ein aktiver, vorprogrammierter Prozess ist, der den für eine Population nachteiligen Effekt des zu langen Überlebens verhindert [5].
Jede dieser Theorien hat für unterschiedliche Teilaspekte des Alterungsprozesses mehr oder weniger Gültigkeit.

Vielleicht erschließt sich der Prozess besser durch eine Sicht auf die primären Leidtragenden, die Gewebe und die sie bildenden Zellen. Ausgehend von Stammzellen werden mitotisch aktive Vorläuferzellen gebildet, welche immer mehr differenzieren und schließlich als terminal differenzierte Zellen ihre Aufgaben im betreffenden Gewebe ausführen (- Abb. 1a). Während die Alterung in den mitotisch sehr aktiven Vorläuferzellen des Darmepithels eher zur vermehrten Tumorentstehung führt, überwiegen in postmitotischen Neuronen degenerative Prozesse. Beide Phänomene werden durch eine Anhäufung zellulärer Schäden verursacht, die dann ein bedrohliches Maß annehmen, wenn sie die Reparaturkapazität der Zellen überschreiten. Dies kann durch eine hohe Anzahl an Schäden pro Zeit geschehen, wie z. B. durch UVLicht induziert beim Sonnenbad, durch Schädigung der Reparaturmechanismen selbst, was auch bei vielen erblichen progeroiden Syndromen zu sehen ist, oder durch Bildung von geschädigten Biomolekülen, mit denen der Reparaturmechanismus nicht umgehen kann, wie z. B. bei Bildung von Alzheimer-Plaques [6]. Die so herausgeforderte Zelle verschafft sich zunächst Zeit, um die Reparatur durchzuführen, im Fall von DNA-Schäden durch Zellzyklusarrest (• Abb. 1). Kann der Schaden nicht repariert werden, bestehen 3 Möglichkeiten:

- die Zelle stirbt ab, klassischerweise durch Apoptose, 


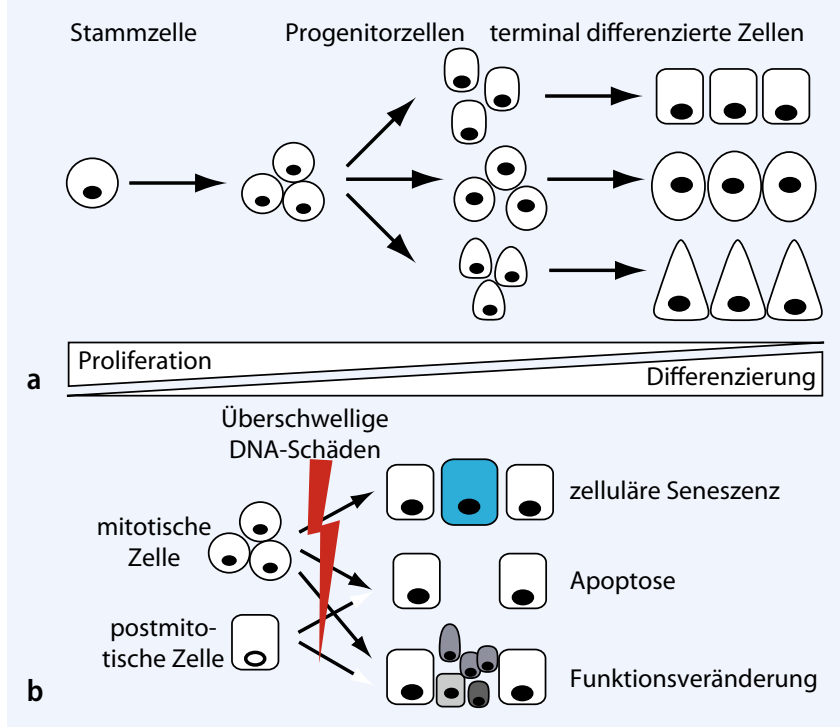

Abb. $1 \Delta$ Auswirkungen von zellulären Schäden auf das Schicksal von mitotischen Vorläuferzellen oder postmitotischen ausdifferenzierten Gewebezellen. a Ausgehend von pluripotenten Stammzellen bilden sich teilungsfähige Vorläuferzellen, deren Tochterzellen dann entsprechend den Anforderungen des jeweiligen Gewebes ausdifferenzieren. $\mathbf{b}$ Je nachdem, in welchem Stadium die Zellen in einem die Kapazität der Reparaturmechanismen übersteigenden Maße geschädigt werden, ergeben sich unterschiedliche Konsequenzen

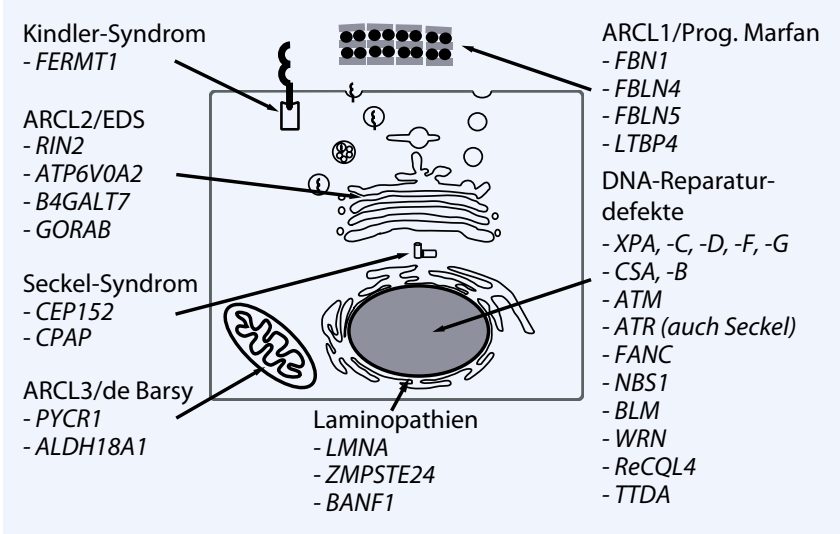

Abb. $2 \Delta$ Zuordnung der Gendefekte segmentaler progeroider Erkrankungen zu den zellulären Strukturen, in denen die Genprodukte ihre Hauptfunktion haben. Beim Kindler-Syndrom liegt der Defekt in einem integrinbindenden Protein, das wahrscheinlich bei der Signaltransduktion eine Rolle spielt. Das progeroide Marfan-Syndrom und die autosomal-rezessive Cutis laxa Typ 1 werden durch Dysfunktion von an der Bildung elastischer Fasern beteiligten mikrofibrillären Proteinen verursacht. Die größte Zahl an Gendefekten lässt sich DNA-Reparaturmechanismen zuordnen, was die Wichtigkeit dieses Prozesses für die Aufrechterhaltung der Gewebehomöostase während des Älterwerdens verdeutlicht. Die Laminopathien führen zu Störungen der Kernlamina. Das De-Barsy-Syndrom und zu seinem Spektrum gehörende Erkrankungen sind wahrscheinlich mit einer Fehlfunktion der Mitochondrien verknüpft. Das Seckel-Syndrom, welches auch durch ATR-Defekte verursacht wird, ist außerdem mit Veränderungen in den zentrosomalen Proteinen CEP250 und CPAP verbunden. Die autosomal-rezessive Cutis laxa Typ 2 wird durch Mutationen in den im Golgi-Apparat lokalisierten Proteinen ATP6V0A2, RIN2 und GORAB verursacht. B4GALT7 ist mit dem progeroiden Ehlers-Danlos-Syndrom verknüpft
- die Zelle verbleibt im Zellzyklusarrest und wird seneszent, oder

- sie lebt weiter, verändert aber ihre Funktion (• Abb. 1b; [7]).

Fatal ist die Variante der Funktionsveränderung mit unkontrollierter Proliferation, vor der sich der Organismus durch Apoptose oder zelluläre Seneszenz schützt. Die untergehenden Zellen können durch Neubildung ersetzt werden, falls nicht die hierfür nötigen Stammzellen betroffen sind. Seneszente Zellen hingegen verbleiben als weitgehend nutzlose Bausteine im Gewebeverband und können akkumulieren, wodurch sie die Funktion des Gewebes stören. Warum kann die Abwehr von unkontrolliert proliferierenden Zellen nicht ausschließlich durch Apoptose erfolgen? Eine Antwort liefert die Tatsache, dass in Tumorzellen, in denen Apoptosemechanismen ausgeschaltet worden sind, häufig zelluläre Seneszenz beobachtet wird und dies somit als zweite Verteidigungslinie fungiert. Außerdem legen neuere Ergebnisse nahe, dass zellulä- re Seneszenz noch andere Funktionen als den Schutz vor Proliferation haben könnte und ihre Aktivierung in diesem Zusammenhang eigentlich pathologisch ist.

\section{\) Etwa 70 segmentale progeroide Syndrome sind derzeit bekannt}

Etwa 70 segmentale progeroide Syndrome sind derzeit bekannt, von denen eine repräsentative Auswahl in den folgenden Artikeln diskutiert werden soll. Wir möchten hier nur eine Übersicht über die generellen Charakteristika und den jeweilig zugrunde liegenden Alterungsmechanismus geben. Eine Übersicht der wichtigsten Genprodukte und ihrer zellulären Lokalisation gibt $\bullet$ Abb. 2.

\section{Auslöser progeroider Erkrankungen}

\section{DNA-Reparaturdefekte}

Der klinische Verlauf des Werner-Syndroms (WS) bildet am ehesten den physiologischen Alterungsprozess - allerdings in beschleunigter Form - ab, da die Betroffenen zunächst eine unauffällige Entwicklung zeigen und der vorzeitige Alterungsprozess erst im 2. Lebensjahrzehnt beginnt. Das defekte Genprodukt ist die Werner-Helikase (WRN), die in verschiedene DNA-Reparaturmechanismen sowie die Aufrechterhaltung der Telomere involviert ist. Wie beschrieben, führen Schäden der DNA und eine Verkürzung der Telomere zum Zellzyklusarrest, gefolgt von zellulärer Seneszenz oder Apoptose. Auch das Bloom- und das Rothmund-ThomsonSyndrom werden durch Defekte an RecQLDNA-Helikasen verursacht [8].

Das Cockayne-Syndrom (CS) beginnt bereits im Kindesalter und ist im Gegensatz zu den vorgenannten Erkrankungen 


\begin{tabular}{|c|c|}
\hline Lokalisation/Art der Erkrankung & Symptome \\
\hline Haut und -anhangsgebilde & $\begin{array}{l}\text { - Pigmentstörungen } \\
\text { - Fibrosierungen der Haut } \\
\text { - Faltenbildung } \\
\text { - Ergrauen der Haare oder Haarverlust } \\
\text { - Änderungen in Menge und Verteilungsmuster des } \\
\text { Fettgewebes }\end{array}$ \\
\hline Muskuloskelettales System & $\begin{array}{l}\text { - Osteoporose } \\
\text { - Degenerative Gelenkveränderungen } \\
\text { - Sarkopenie }\end{array}$ \\
\hline $\begin{array}{l}\text { Gehirn, peripheres Nervensystem } \\
\text { und Sinnesorgane }\end{array}$ & $\begin{array}{l}\text { - Demenz oder degenerative neuropathologische Symptome } \\
\text { - Polyneuropathie } \\
\text { - Katarakt } \\
\text { - Makuladegeneration } \\
\text { - Schwerhörigkeit }\end{array}$ \\
\hline Kardiovaskuläres System & $\begin{array}{l}\text { - Arteriosklerose } \\
\text { - Herzinsuffizienz }\end{array}$ \\
\hline $\begin{array}{l}\text { Endokrines System und Meta- } \\
\text { bolismus }\end{array}$ & $\begin{array}{l}\text { - Diabetes mellitus } \\
\text { - Störungen des Lipidstoffwechsels } \\
\text { - Hypogonadismus }\end{array}$ \\
\hline Maligne Erkrankungen & - Für höheres Lebensalter typische Tumoren \\
\hline
\end{tabular}

durch eine deutliche neurodegenerative Komponente und eine leicht erhöhte UVEmpfindlichkeit der Haut gekennzeichnet. Ein erhöhtes Risiko für Tumorentwicklung besteht nicht. Hingegen zeigen Patienten mit Xeroderma pigmentosum (XP) eine extrem erhöhte UV-Empfindlichkeit der Haut, die zu altershautartigen Veränderungen und Hautkrebs führt (- Tab. 1; [9]). Beide Erkrankungen werden durch Defekte in der Nukleotidexzisionsreparatur verursacht.

Schließlich sind in dieser Gruppe der progeroiden Erkrankungen auch noch die Fanconi-Anämie (FA) und die Ataxia teleangiectatica (AT) zu erwähnen, welche u. a. eine fehlende Aktivierung der DNAReparaturmechanismen zeigen. Von den 3 Krankheitsgenen für das Seckel-Syndrom, welches mit beschleunigter Alterung bestimmter Gewebe in der 2.-3. Lebensdekade einhergehen kann, spielt eines, ATR, ebenfalls eine Rolle bei der Induktion der DNA-Reparatur. Für CEP152 konnte zusätzlich zur zentrosomalen Funktion eine Beteiligung an der ATRvermittelten Zellzyklusregulation nachgewiesen werden, während $C P A P$ eine zentrosomale Funktion hat [10]. Den meisten der hier erwähnten Erkrankungen ist ein primärer Defekt von DNA-Reparaturmechanismen gemeinsam. Aufgrund der Wichtigkeit dieses Mechanismus für die Alterung ist ihm ein eigener Beitrag ge- widmet. Anhand des Gesagten lässt sich mulation von DNA-Schäden kommt, auf die die Zellen mit Apoptose, zellulärer Seneszenz oder maligner Entartung reagieren. Durch Einbeziehung von Stammzellen nimmt außerdem die Fähigkeit ab, abgestorbene Zellen zu ersetzen.

Geprägt wurde der Begriff der Progerie im Zusammenhang mit der Beschreibung des Hutchinson-Gilford-Progerie-Syndroms (HGPS), bei dem der Alterungsprozess im Gegensatz zum Werner-Syndrom bereits in der frühen Kindheit beginnt [11]. Hier ruft die ursächliche Mutation eine spezifische Variante des LaminA/C-Proteins hervor, welche Progerin genannt wird und verschiedene Auswirkungen hat, u. a. eine erhöhte Produktion von Sauerstoffradikalen und eine Verkürzung der Telomere [12]. Aus diesem Grund stellt das HGPS innerhalb der Gruppe der progeroiden Erkrankungen durch defekte DNA-Reparatur einen Sonderfall dar. Eine überlappende Krankheitsentität geht auf Veränderungen im ZMPSTE24-Gen zurück. Das Genprodukt ist verantwortlich für das Prozessieren von Lamin A/C [13]. Mutationen in $B A N F 1$, welches für ein mit Lamin A/C interagierendes Protein kodiert, erzeugen einen sehr spezielableiten, dass es hierdurch zu einer Akku-

\section{Defekte Kernlamina}

len progeroiden Phänotyp mit Osteolysen an den Kieferknochen, Skoliose und starker Osteoporose, auch Néstor-GuillermoSyndrom genannt [14].

\section{Veränderungen an extrazellulärer Matrix, Golgi-Apparat und Mitochondrien}

Neben der Neigung zur Blasenbildung zeigt die Haut von Patienten mit KindlerSyndrom auch erhöhte UV-Empfindlichkeit sowie eine atrophe Altershaut mit Poikilodermie in Kombination mit starker Parodontose [15]. Zwar ist eine Akkumulation von DNA-Schäden bei dieser Erkrankung noch nicht nachgewiesen worden, aber die Ähnlichkeiten zum Hautphänotyp von einigen Erkrankungen mit DNA-Reparaturdefekt weisen in diese Richtung. Eines der defekten Genprodukte, Kindlin, spielt eine Rolle bei der integrinvermittelten Adhäsion und Signaltransduktion [16].

Primär ein Protein der extrazellulären Matrix, Fibrillin 1, ist beim progeroiden Marfan-Syndrom betroffen, bei dem der vorgealterte Aspekt v. a. auf eine starke Lipodystrophie zurückgeht [17]. Ebenso durch eine starke Beteiligung der extrazellulären Matrix gekennzeichnet sind die Cutis-laxa-Syndrome, von denen v. a. die autosomal-rezessive Cutis laxa (ARCL) progeroide Züge zeigt. Während ARCL Typ 1 durch Mutationen in ECMProteinen (FBLN4, FBLN5, LTBP4) verursacht wird, sind bei ARCL Typ 2 und Typ 3 intrazelluläre Proteine mit vorwiegender Rolle in Endosomen (RIN2), Golgi-Kompartiment (ATP6V0A2, GORAB) und Mitochondrien (ALDH18A1, PYCR1) betroffen [18]. Die progeroide Form des Ehlers-Danlos-Syndroms ist ebenfalls mit einem Defekt in einem Golgi-Protein (B4GALT7) verbunden, das in der Glykosaminoglykansynthese eine Rolle spielt [19]. Der genaue Mechanismus, durch den diese Gendefekte zu progeroiden Symptomen führen, ist noch nicht aufgeklärt.

Die wichtigsten segmentalen progeroiden Syndrome, für die noch kein Gen gefunden werden konnte, sind das GAPO-Syndrom, das Hallermann-Streiffund das Wiedemann-RautenstrauchSyndrom [20-22]. 


\section{Korrespondenzadresse}

\section{Prof. Dr. rer. nat. U. Kornak}

Institut für Medizinische Genetik

und Humangenetik

Charité - Universitätsmedizin Berlin

Augustenburger Platz 1, 13353 Berlin

uwe.kornak@charite.de

Interessenkonflikt. Der korrespondierende Auto gibt für sich und seine Koautoren an, dass kein Interessenkonflikt besteht

\section{Literatur}

1. Martin GM (2005) Genetic modulation of senescent phenotypes in Homo sapiens. Cell 120(4):523-532

2. Burtner CR, Kennedy BK (2010) Progeria syndromes and ageing: what is the connection? Nat Rev Mol Cell Biol 11(8):567-578

3. Dawkins R (1976) The Selfish Gene. Oxford University Press

4. Kirkwood TB, Holliday R (1979) The evolution of ageing and longevity. Proc R Soc Lond B Biol Sci 205(1161):531-546

5. Kirkwood TB, Melov S (2011) On the programmed/ non-programmed nature of ageing within the life history. Curr Biol 21(18):R701-707

6. Campisi J, Vijg J (2009) Does damage to DNA and other macromolecules play a role in aging? If so, how? J Gerontol A Biol Sci Med Sci 64(2):175-178

7. Campisi J, d'Adda di Fagagna F (2007) Cellular senescence: when bad things happen to good cells. Nat Rev Mol Cell Biol 8(9):729-740

8. Seki M et al. (2008) RecQ family helicases in genome stability: lessons from gene disruption studies in DT40 cells. Cell Cycle 7(16):2472-2478

9. Cleaver JE (2005) Cancer in xeroderma pigmentosum and related disorders of DNA repair. Nat Rev Cancer 5(7):564-573

10. Klingseisen A, Jackson AP (2011) Mechanisms and pathways of growth failure in primordial dwarfism. Genes Dev 25(19):2011-2024

11. Hennekam RC (2006) Hutchinson-Gilford progeria syndrome: review of the phenotype. Am J Med Genet A 140(23):2603-2624

12. Huang $S$ et al (2008) Accelerated telomere shortening and replicative senescence in human fibroblasts overexpressing mutant and wild-type lamin A. Exp Cell Res 314(1):82-91

13. Shackleton S et al (2005) Compound heterozygous ZMPSTE24 mutations reduce prelamin A processing and result in a severe progeroid phenotype. J Med Genet 42(6):e36

14. Puente XS et al (2011) Exome sequencing and functional analysis identifies BANF1 mutation as the cause of a hereditary progeroid syndrome. Am J Hum Genet 88(5):650-656

15. Has C et al (2011) Kindler syndrome: extension of FERMT1 mutational spectrum and natural history. Hum Mutat 32(11):1204-1212

16. Larjava H, Plow EF, Wu C (2008) Kindlins: essential regulators of integrin signalling and cell-matrix adhesion. EMBO Rep 9(12):1203-1208

17. Graul-Neumann LM et al (2010) Marfan syndrome with neonatal progeroid syndrome-like lipodystrophy associated with a novel frameshift mutation at the $3^{\prime}$ terminus of the FBN1-gene. Am J Med Genet A 152A(11):2749-2755
18. Morava E et al (2009) Autosomal recessive cutis laxa syndrome revisited. Eur J Hum Genet 17(9):1099-1110

19. Quentin E et al (1990) A genetic defect in the biosynthesis of dermatan sulfate proteoglycan: galactosyltransferase I deficiency in fibroblasts from a patient with a progeroid syndrome. Proc Natl Acad Sci U S A 87(4):1342-1346

20. Pivnick EK et al (2000) Neonatal progeroid (Wiedemann-Rautenstrauch) syndrome: report of five new cases and review. Am J Med Genet 90(2):131140

21. Cohen MM Jr (1991) Hallermann-Streiff syndrome: a review. Am J Med Genet 41(4):488-499

22. Nanda A et al (2010) GAPO syndrome: a report of two siblings and a review of literature. Pediatr Dermatol 27(2):156-161 